Aksaray University
Journal of Science and Engineering
e-ISSN: 2587-1277
http://dergipark.gov.tr/asujse
http://asujse.aksaray.edu.tr

Research Article

\title{
Detection of Tumor Slice in Brain Magnetic Resonance Images by Feature Optimized Transfer Learning
}

\author{
Salih Celik, Omer Kasim* \\ Dumlupınar University, Faculty of Technology, Electrical Engineering Department, Kutahya, Turkey.
}

\begin{tabular}{|c|c|c|c|}
\hline -Received Date: Nov 03, 2020 & -Revised Date: Dec 08, 2020 & -Accepted Date: Dec 29, 2020 & -Published Online: Dec 29, 2020 \\
\hline
\end{tabular}

\begin{abstract}
This study includes investigating the presence of tumor regions in Magnetic Resonance Imaging (MRI) slices. Since the MRI taken from a patient consists of many slices, it may take time for experts to review these images. The aim of the study is to evaluate the specialist's MRI slices more quickly. The image of each MRI slice taken from the patient was applied to the Alexnet transfer learning algorithm and the properties of the image were obtained. These features are optimized with the Relieff feature selection algorithm to achieve optimum success. The highest accuracy has been achieved with the support vector machine classifier, in which optimized features are used. The study was validated with 3 different combinations by training with two datasets and testing with the other. Thus, a method that can work under different conditions were obtained. The performance metrics of the study were obtained by taking the average of the successes obtained from each data set. MRIs were trained with Alexnet transfer learning model and performance analysis was performed on the obtained classification models. The feature optimization used both increased the success to $97.55 \%$ and reduced the processing time from 0.4064 to 0.3045 seconds. The proposed model with a high success rate and a rapid classification is expected to assist the expert in both diagnosis and treatment planning.
\end{abstract}

\section{Keywords}

Magnetic Resonance Imaging, Feature Extraction, Alexnet Transfer Learning, Relieff Feature Selection, Support Vector Machines

*Corresponding Author: Omer Kasim, omer.kasim@dpu.edu.tr, 1 0000-0003-4021-5412 


\section{INTRODUCTION}

Computer Aided Detection (CAD) enables information communication devices to be used by experts in diagnosis and treatment. One of the application areas of CAD is the detection of brain tumors with Magnetic Resonance Imaging (MRI) [1]. MRIs are widely used to identify existing diseases and to plan treatment. They provide structural and contextual information about tumor cells, which can affect the vital functions of the patient.

MRIs are taken in slices. In manual detection, these slices are evaluated by experts. In this examination, experts diagnose the tumors in the image and plan the treatment according to the type and size of the tumor. Accordingly, the duration of diagnosis and the accuracy of classification of the tumorous slices depends on the knowledge and experience of the specialist. Furthermore, manual examination and feature extraction is problematic in terms of early and reliable diagnosis [2]. At the same time, manual examination is a significant waste of time for experts. Therefore, it is important to detect and classify the tumor in the MRI slice by analyzing them fully automatically to assist experts detect brain tumors. In this schema, the tumor in MRI slice can be detected accurately in short time. Thus, it is aimed to contribute the early diagnosis process by saving time in tumor diagnosis by eliminating unnoticed MRI slices. Feature extraction and classification are essential in automated processing of MRIs [3]. It is the motivation of the study that transfer learning methods give more successful results than other statistics and measurement methods from the extraction of the features of the images.

Many studies have been conducted in the literature in this field in recent years with this motivation. Kaur et al. evaluated their model in 3 different data sets with the deep Convolutioanl Neural Network (CNN) model they proposed. The authors achieved 100\%, 94\% and 95.92\% success in Harvard, clinical, and benchmark Figshare repository datasets, respectively. In addition, many transfer learning has been tried and Alexnet has been identified as the most successful model [4]. Praveen et al. proposed a multi-step approach for tumor detection from MRI. The first step of this approach involved image filtering. This process included image cropping, scaling and histogram equalization methods, respectively. Then, the gray level concurrence matrix method was used to extract MRI features. After the pretreatment step, the images with tumors were classified using the Random Forest method. 120 patient data were used in the evaluation and the classification accuracy of the developed model was found to be $87.62 \%$ [5]. Havaei et al. used Brats2013 data set to classify the MRIs with cascading TwoWay CNN. These CNNs provided local and global processing simultaneously with the data set. The proposed method has achieved $88 \%$ successful tumorous MRIs [6]. Dimilliler and İlhan 
proposed a multi-layer perceptron classification system of brain MRIs as healthy or tumor. First, brain MR images of $256 \times 256$ were resized to $64 \times 64$ in order to complete the training in less time and pre-processed with histogram equalization method. From the pre-processed brain MR images, tumorous images and tumor-free images were labeled by artificial neural network (ANN). The classification success was achieved as 90\% [7]. Kazdal et al. classified 497 MRI slices with $84.26 \%$ tumor and no tumor using a rule-based CAD technique based on morphological features [8]. Muhammed Nazir et al. first filtered brain MRIs and cleaned them from noise. Then, the authors obtained the features of each image on average color moment. These features were classified by an ANN. The authors obtained 91.8\% accuracy [9]. Dawud et al. developed a brain hemorrhage detection from MRIs. The authors extracted features with the Alexnet model and the extracted features are applied to the SVM classifier. The classification accuracy of the method was found to be $93.48 \%$ [10]. Bahadure et al. applied the properties they obtained with the neuro-fuzzy inference system to the $\mathrm{CNN}$ and Genetic Algorithm [11]. Sajjad et al. proposed a detection system to obtain tumorous MRI slices. Firstly the tumor region was selected by segmentation from MRIs. Then, the classification process was carried out with the CNN model. Classification success was achieved as $94.58 \%$ [12]. Tajik et al. proposed a hybrid system in his study. The MRIs were classified by using genetic algorithm and GLCM features with K-Nearest Neighbor classifier. The performance of the hybrid system is calculated as $96.67 \%$ [13]. Toğaçar et al. proposed a new unified convolutional neural network $(\mathrm{CNN})$ model. The features obtained by AlexNet and VGG-16 transfer learning models were applied to the recursive feature removal (RFE) algorithm and the new features obtained are classified with SVM. The success rate obtained in this study is 96.77\% [14]. Ari et al. used extreme learning machine local receptive algorithm and alexnet model to classify tumorous area from MRI. The authors achieved 97.18\% success with ELM-LRF and 96.91\% success with AlexNet [15].

In the studies suggested in the literature, feature extraction with transfer learning and tumor MRI classification, classification of features from machine learning and deep learning architecture, and classification methods by reducing features are presented. These studies were generally validated with a single data set. Unlike other studies, the feature vectors obtained with the Alexnet transfer learning model were classified by SVM method in 3 different data sets in this study. The data set of the study was obtained using Rembrandt [16], Rider Neuro MR [17] and Brain Tumor Progression [18, 19]. The data set of the study was created with 1800 axial healthy MRI slices from 170 patients from these data sets. Performance analysis was performed 
to obtain the most successful model in determining tumor and non-tumor MRI slices with this data set. In the training of the models, two different data sets were combined and 1200 images were obtained and verified by cross-validation method. The third data set was used for testing. Thus, a robust method that can work under different conditions has been obtained. It was determined as the system in which Alexnet, Relieff and SVM are used together. In this classification system, which was created by integrating Relieff feature selection algorithm into Alexnet and SVM models, the test success rate was $97.55 \%$; classification time was obtained as 0.3045 seconds.

The contributions of the study can be listed below.

- The high success rate obtained by using 3 different data sets together shows that the proposed method can work successfully on data sets with different characteristics. Thus, a more robust system assistant for experts was obtained.

- The proposed method can help experts reduce their busy schedule and assist them in identifying tumors that might be overlooked at early diagnosis.

- The accuracy of SVM classifier is increased and a more effective system is achieved by ensuring its performance with the Relieff feature optimization.

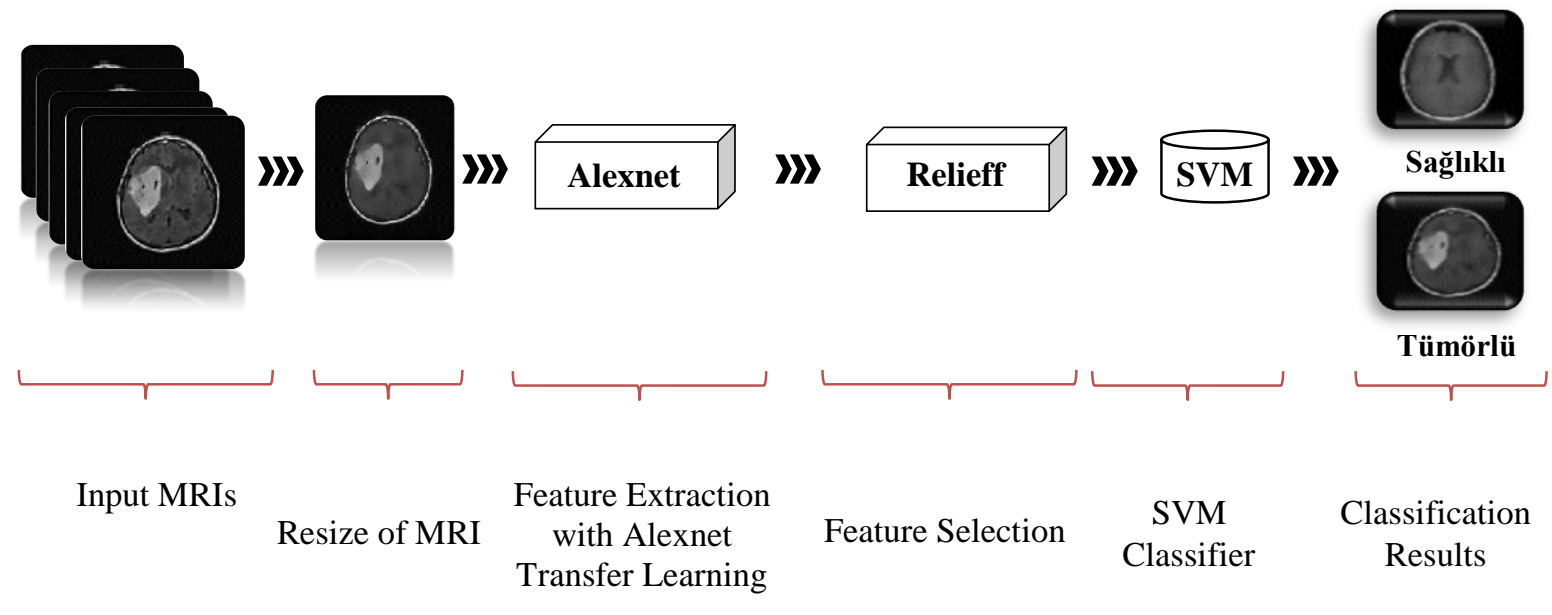

Figure 1. Flow diagram of the proposed model.

\section{MATERIAL AND METHODS}

The data set containing MR images is first converted into a format and size suitable for network structures. Firstly, MR images in DICOM format were converted to JPEG format. Then, each MR image slice was resized to $256 \times 256$. Images in the data set have 3-dimensional depth in RGB format. Then, the new format and size images obtained were used as input data in the 
Alexnet model. The Alexnet transfer learning algorithm was used to extract the features in the image to determine the tumors in the MRI slices proposed in the study. Relieff algorithm was used for optimization of the properties obtained by transfer learning. The features reduced by this algorithm are classified with SVM classifier and MRI slices with tumors are detected. The flow chart of the study is given in Fig. 1.

\subsection{Alexnet Transfer Learning Model}

Deep learning is a machine learning technique that is inspired by the human brain that has the ability to analyze, observe, make decisions for complex problems and process them in a short time. It has taken its place in academic studies as an application that can extract features with or without supervision, can perform operations such as transformation and classification by using large amounts of data and is frequently used in today's technology.

AlexNet is the first large-scale deep network capable of image classification. This architecture is generally one of those architectures that move away from stacking convolution and pooling layers on top of each other in a consecutive structure. This is because stacking all layers and adding multiple filters costs a calculation and memory and increases the possibility of memorization. Alexnet has used modules connected in parallel to overcome this situation [20]. Network architecture of AlexNet is given in Fig. 2.

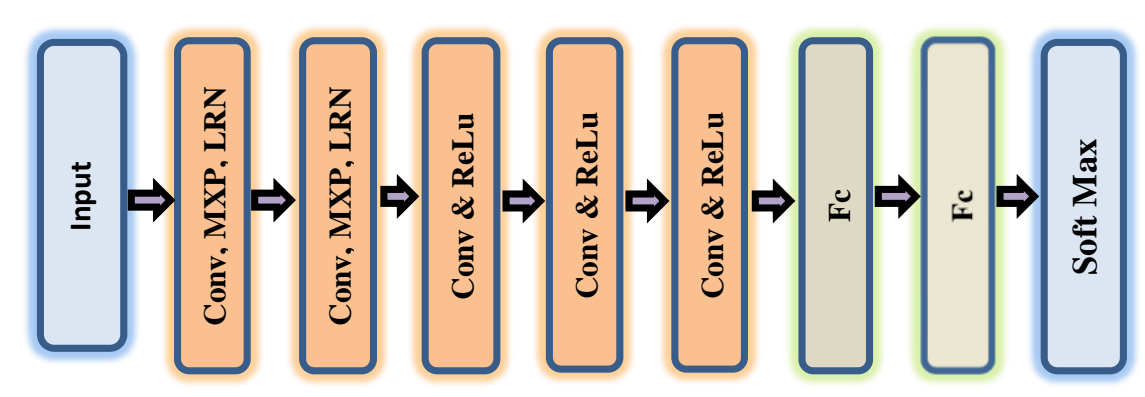

Figure 2. AlexNet Architecture

The Alexnet architecture is previously trained with millions of images and has the ability to classify in 100 object categories. Before the softmax layer, the network operation was interrupted and it was used to extract the properties of the images of brain MRIs.

\subsection{Relieff Feature Selection Algorithm}

Feature selection is a subset of features belonging to a class and obtained by feature extraction models. The relieff feature selection algorithm, with filter type feature selection, gives the best result in estimating the feature significance for models that use double distances between observations to predict the response, directing the distance [21]. 
When $\mathrm{X}$ input vector is applied to the algorithm that iterates, vectors with the least Euclidean distance from a class to $X_{a}$ are obtained. The nearest samples of the same class $\left(\mathrm{nH}_{\mathrm{a}}\right.$ and $\left.\mathrm{nM}_{\mathrm{a}}\right)$ are obtained and the weight vector is updated with Equation 1 and iteration is continued.

$$
W_{a}=W_{a}-\left(X_{a}-n H_{a}\right)^{2}+\left(X_{a}-n M_{a}\right)^{2}
$$

The weight of a particular feature at the end of each iteration decreases if it differs more than close samples of the same class, or vice versa with Equation 1. When the iterations are over, properties that are greater than a certain threshold value are selected [22].

\subsection{SVM Classifier}

SVM classifier is very successful and one of the most used methods in classification problems. The algorithm works by separating points on the plane by certain boundary lines. Basically the point in a plane is given as in Equation 2.

$$
D=\left\{\left(x_{i}, c_{i}\right) \mid x_{i} \in R^{p}, c_{i} \in\{-1,1\}\right\}_{i=1}^{n}
$$

In Equation 2, $c_{i}$ refers to the class labels. These values can be either -1 or +1 . The training data $\mathrm{D}$ is consisted of $\mathrm{n}$ elements. It is any sample in $\mathrm{n}$-dimensional space. Equation 2 is used for training SVM.

$$
\boldsymbol{w} \boldsymbol{x}-\boldsymbol{b}=\mathbf{0}
$$

In Equation 3, w is the normal of the decision function, $x$ is the points on this line, and $b$ is the trend value. The aim here is to obtain $w$ and $b$ with the help of training data. This equation can also be thought of as the classical line equation $a x+b$. The distance between the two groups is $\mathrm{b} /\|\mathrm{w}\|$ expressed in the form. Then, the equations were obtained as a result of the operations that help to obtain the boundary common line between the lines by shifting them. SVMs are classifiers that do not have an estimate or information about the distribution and do not take parameters. In training sets, inputs and outputs are matched to obtain decision functions that will classify input data [23]. It is widely used in classification problems because of its lack of congruence problems, its ability to work with more than one independent variable, and its success in modeling complex boundaries.

\section{RESULTS AND DISCUSSIONS}

The data set of the study was obtained from Rembrandt [16], Rider Neuro [17] and Brain Tumor Progression (BTP) $[18,19]$ MRI data sets, which are used in the literature and whose reliability has been proven by scientific studies, which are in the National Institutes of Health (NIH) Cancer Imaging Archive (TCIA). Each of the images in the sets consists of axial region brain MRIs. Sets and their contents are as shown in Table 1. 
Table 1. The data set of the study.

\begin{tabular}{lcccc}
\hline Dataset & Content & $\begin{array}{c}\text { Number of } \\
\text { patients }\end{array}$ & $\begin{array}{c}\text { Number of Tumorous } \\
\text { MRI Slice }\end{array}$ & $\begin{array}{c}\text { Number of without } \\
\text { Tumorous MRI Slice }\end{array}$ \\
\hline Rembrandt & MRI & 130 & 300 & 300 \\
Brain Tumor Progression (BTP) & MRI & 20 & 310 & 290 \\
Rider Neuro MRI & MRI & 19 & 320 & 280 \\
\hline
\end{tabular}

Three different data sets were created in the study by separating the tumorous MRI slice and without tumorous MRI slice shown in Table 1 with the help of an expert. The data sets, which were formed as a double group, were divided into two different groups, healthy and tumor.

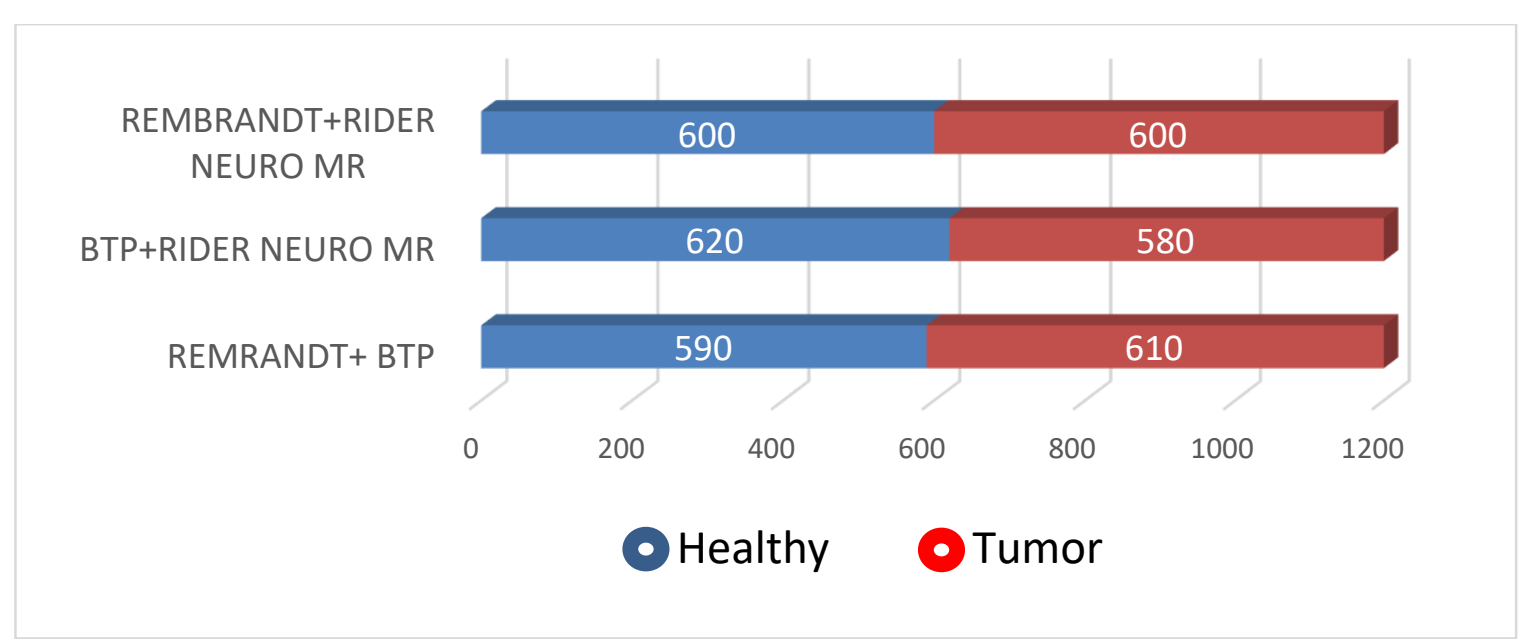

Figure 3. Three different datasets and their contents.

Three different data sets were used in the study, as shown in Figure 3. The first group consists of 50\% healthy MRI data and 50\% tumor MRI data of Remrandt and Rider Neuro MR data set. The second group consists of $51.6 \%$ healthy and $48.4 \%$ tumor MRI data of the BTP and Rider Neuro MR data set. The third group of Rembrandt and BTP data set consists of MRI data of $49.2 \%$ healthy and $50.8 \%$ tumorous. In order to avoid the disadvantage of the unbalanced dataset, the dataset was organized by taking approximately equal numbers of normal and tumor images.

\subsection{Training Alexnet Transfer Learning Model}

Transfer learning architectures are generally used in classification and feature detection applications. It has two basic functions: convolution and pool. The convolution and the arrangement of the pool layers continue until high accuracy is achieved. In addition, some feature layers have feature maps. Learning different characteristics of the network. It is achieved by keeping the traceable parameters by sharing the weights in the feature maps among themselves. Deep learning architectures can learn almost all the acquired features. The Alexnet deep learning architecture used in the study was trained using three different data sets. 
Feature vectors created by obtaining attributes belonging to healthy and tumor classes using Alexnet deep learning architecture. It was optimized with the Relieff feature selection algorithm and applied to the SVM classifier and MRI slices were classified as tumor or healthy.

\subsection{Evaluation of the Proposed Method}

In this study, Alexnet model is used as a transfer learning architecture. Three different data sets, Rembrandt, BTP and Rider Neuro MR, were used for training and testing. 600 pieces of MR images included in each data set were used for training and verification data in pairs for classification models. In the experimental sets, 960 MRIs were used for training the model and 240 MRIs were used for validation. 240 MRI slice images not used in training and validation were used to test the proposed model. MRIs, whose attributes of healthy and tumor classes were obtained with the Alexnet transfer learning algorithm, were classified by SVM method as a classification process. The most successful network has been determined among 6 different classification algorithms.

Experiments were conducted by creating training sets in binary combinations for Rembrandt, Brain Tumor Progression and Rider Neuro MR data sets. Each data set created contains 1200 axial region healthy and tumor brain images. The Alexnet transfer learning algorithm was optimized with the Relieff feature selection algorithm, and the success rates and test times were compared and the performance metrics of 3 datasets, average success of 3 models and average test times are given.

In the experiments of the study, confusion matrixes obtained after testing the model trained with Rembrandt and Rider Neuro MRI data sets with 240 BTP data sets were given as the second data set. The classification performances of different transfer learning models are compared. According to the results, the most successful model was determined as the Alexnet $($ Relieff + SVM) model.

According to the test results, the most successful model was determined as the Alexnet (Relieff + SVM) model. Cross-validation accuracy and test accuracy of six different studies are given in Table 2.

According to the complexity matrices obtained with the experimental results, performance analysis was made between 3 data set combinations and six different classification models. As a result of the analysis made. The hybrid model obtained by adding the Relieff feature selection algorithm to the Alexnet deep learning model, which was trained using the Rembrandt data set, was the most successful model. 
Table 2. Verification values and test durations of experimental results obtained with Rembrandt, BTP and Rider Neuro MR data set.

\begin{tabular}{llcccc}
\hline Dataset & \multicolumn{1}{c}{ Model } & $\begin{array}{c}\text { Hold-out } \\
\text { Rate }\end{array}$ & $\begin{array}{c}\text { Cross- } \\
\text { validation } \\
\text { Rate }\end{array}$ & $\begin{array}{c}\text { Test } \\
\text { accuracy } \\
\text { (\%) }\end{array}$ & $\begin{array}{c}\text { Test } \\
\text { performance } \\
\text { (Seconds) }\end{array}$ \\
\hline Rembrandt & Alexnet(SVM) & 0.9590 & 0.9481 & $95.10 \%$ & 0.3204 \\
& Alexnet(Relieff+SVM) & 0.9754 & 0.9757 & $98.40 \%$ & 0.2851 \\
\hline \multirow{2}{*}{ BTP } & Alexnet(SVM) & 0.9486 & 0.9309 & $95.08 \%$ & 0.5769 \\
& Alexnet(Relieff+SVM) & 0.9672 & 0.9599 & $96.72 \%$ & 0.3145 \\
\hline \multirow{2}{*}{ Rider Neuro } & Alexnet(SVM) & 0.9508 & 0.9563 & $96.72 \%$ & 0.3219 \\
& Alexnet(Relieff+SVM) & 0.9672 & 0.9399 & $97.54 \%$ & 0.3141 \\
\hline $\begin{array}{l}\text { Average of } \\
\text { Performance } \\
\text { Metrics }\end{array}$ & Alexnet(SVM) & 0.9528 & 0.9451 & $95.63 \%$ & 0.4064 \\
\hline
\end{tabular}

In addition, when the test times of the classification algorithms given in Table 2 are compared, the model obtained by optimizing Alexnet with Relieff, which is the study using the Rembrandt data set, was determined as the fastest model. The average accuracy of proposed method is obtained as $97.55 \%$. The time taken for the test was obtained as 0.3045 seconds.

In Figure 4, the variables obtained from the complexity matrix of the most successful model and their values are given.

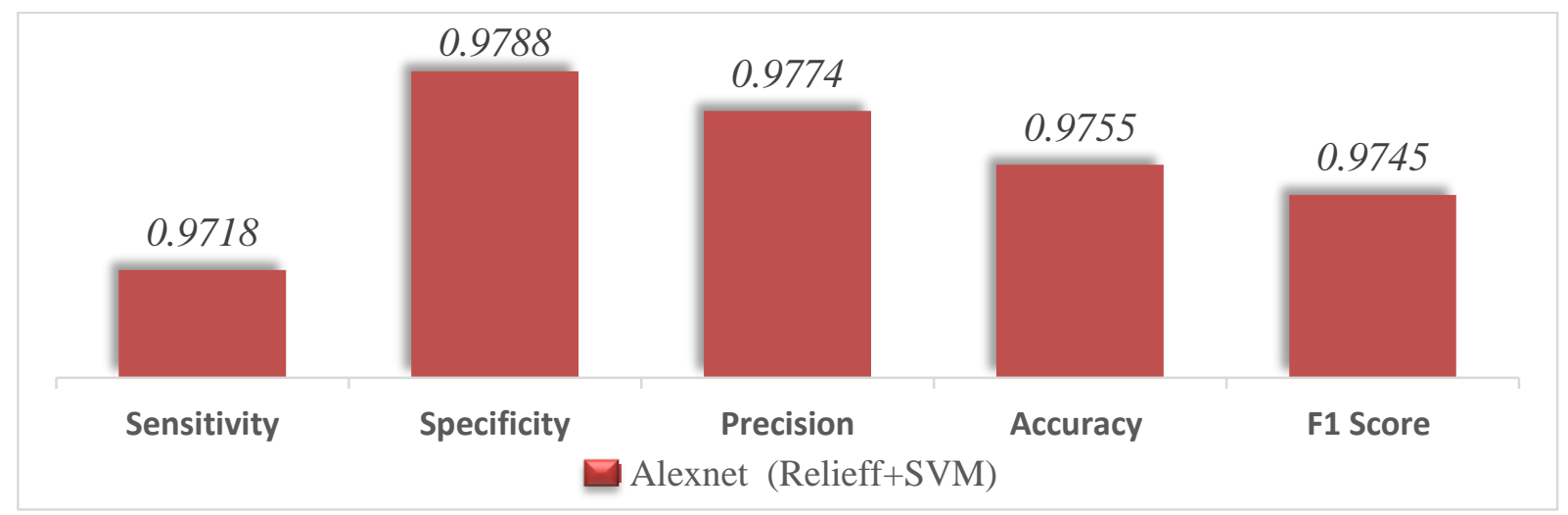

Figure 4. Performance metrics values derived from the complexity matrix of the most successful system.

As given in Figure 4, especially when considering the commonly used F1 score to represent the classification achievements; The Alexnet and Relieff model has been demonstrated as the most successful model. Moreover, the performance rates of the variables of sensitivity, specificity, sensitivity and accuracy have very high values.

\subsection{Comparison with the State-of-the-Art Methods}

The proposed model and deep learning studies conducted in the literature and their success rates are given in Table 5. The CAD studies about detection of tumorous brain MRIs are expressed 
that similar methods and datasets. The accuracy of proposed method is gathered similar to or better than other methods.

Table 5. Studies in the literature and success rates.

\begin{tabular}{|c|c|c|c|}
\hline Studies & Method & Database & Accuracy \\
\hline Ari et al. [24] & Morphological Analysis with kNN & Rembrandt & $87.52 \%$ \\
\hline Ghasemi et al. [25] & Gradient Descent with KSVD & Rembrandt & $98.31 \%$ \\
\hline Amin et al. [26] & Linear SVM Kernel & Rider Neuro & $90 \%$ \\
\hline Doğan et al. [27] & Meta-Learner, kNN & Rembrandt & $96.93 \%$ \\
\hline $\begin{array}{l}\text { Avarage accuracy } \\
\text { of proposed method } \\
\text { in } 3 \text { dataset }\end{array}$ & Alexnet, Relieff and SVM. & $\begin{array}{c}\text { Rider Neuro, } \\
\text { Rembranndt, } \\
\text { BRP }\end{array}$ & $97.55 \%$ \\
\hline
\end{tabular}

In similar studies using the datasets used in the study, Ghasmi et al using Rembrandt achieved 98.31\% success [25]. Amin et al achieved 90\% success with linear SVM for the Rider Neuro data set [26].

Along with the experimental results; The hybrid system, in which the Alexnet deep learning model and the Relieff feature selection algorithm are used together, has been identified as the most successful model in terms of performance and speed in general. The average accuracy of the proposed method in the experiments performed was $97.55 \%$. The time taken for the test was obtained as 0.3045 seconds. The ROC curve of the most successful system is given in Fig. 5.

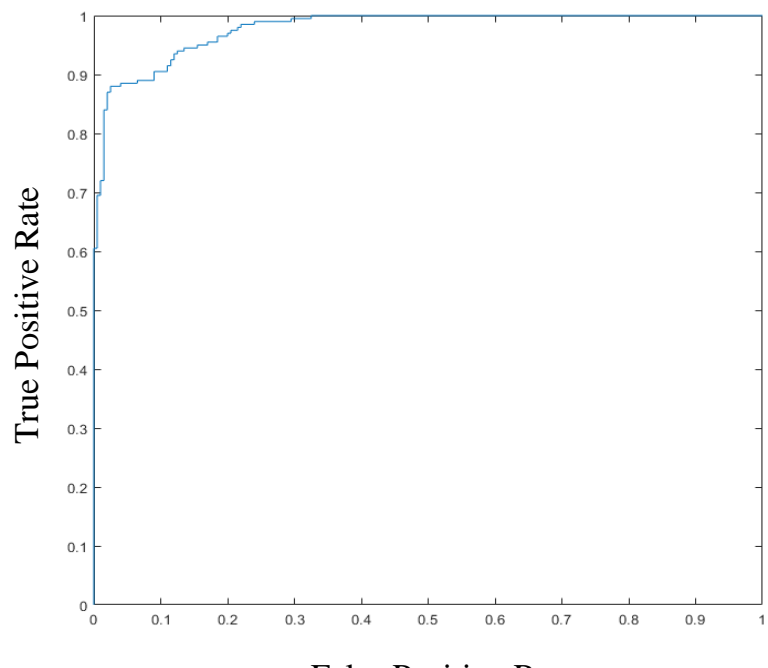

False Positive Rate

Figure 5. ROC curve of Alexnet (Relieff + SVM) system, which is the most successful model.

The ROC Curve describes the relative differences between the sensitivity and precision of the detection system of the tumor brain MRI slice. 


\section{CONCLUSION}

In this study, an automated system for classifying brain cancer MRI slices using Magnetic Resonance Imaging (MRI) taken from 3 different datasets is presented. The test of the proposed method is done at the level of classifying SVM and the other dataset by optimizing the features from Alexnet transfer learning, which is trained with images taken from 2 different datasets, with the Relieff method. Accuracy, sensitivity, specificity, sensitivity and f1 score performance metrics are used to evaluate the proposed method. In the experiments, it was determined that feature optimization increases the success and shortens the working time. The proposed methodology can be used as a helpful tool for busy professionals to examine MRI slices with tumor detection only, rather than whole MRI slices. The results of the experiments reveal the superior performance of the proposed model compared to previous methods. This study will help to examine brain tumors accurately on MRI slices.

\section{References}

[1] G.S. Tandel, M. Biswas, O.G. Kakde, A. Tiwari, H.S. Suri, M. Turk, B.K. Madhusudhan, A review on a deep learning perspective in brain cancer classification, Cancers, 11:1 (2019) 111.

[2] H. Mohsen, E.S.A. El-Dahshan, E.S.M. El-Horbaty, A.B.M. Salem, Classification using deep learning neural networks for brain tumors, Future Computing and Informatics Journal, 3:1 (2018) 68-71.

[3] Y. Yang, L.F. Yan, X. Zhang, Y. Han, H.Y. Nan, Y.C. Hu, X.W. Ge, Glioma grading on conventional MR images: a deep learning study with transfer learning, Frontiers in neuroscience, 12 (2018) 804.

[4] T. Kaur, T.K. Gandhi, Deep convolutional neural networks with transfer learning for automated brain image classification, Machine Vision and Applications, 31 (2020) 1-16.

[5] G.B. Praveen, A. Agrawal, Multi stage classification and segmentation of brain tumor, 3rd International Conference on Computing for Sustainable Global Development (2016).

[6] M. Havaei, A. Davy, W.D. Farley, A. Biard, A. Courville, Y. Bengio, C. Pal, P.M. Jadoin, H. Larochelle, Brain tumor segmentation with deep neural networks, Medical Image Analysis, (2016).

[7] K. Dimililer, A. Ilhan, Effect of Image Enhancement on MRI Brain Images with Neural Networks, Procedia Computer Science, 102 (2016) 39-44.

[8] S. Kazdal, B. Doğan, A.Y. Çamurcu, Computer-aided detection of brain tumors using image processing techniques 23nd Signal Processing and Communications Applications Conference, (2015) 863-866.

[9] M. Nazir, F. Wahid, S.A. Khan, A simple and intelligent approach for brain MRI classification, Journal of Intelligent \& Fuzzy Systems, 28:3 (2015) 1127-1135.

[10] A.M. Dawud, K. Yurtkan, H. Oztoprak, Application of Deep Learning in Neuroradiology: Brain Haemorrhage Classification Using Transfer Learning, Computational Intelligence and Neuroscience, (2019). 
[11] N.B. Bahadure, A.K. Ray, H.P. Thethi, Comparative approach of MRI-based brain tumor segmentation and classification using genetic algorithm. Journal of digital imaging, 31:4 (2018) 477-489.

[12] M. Sajjada, S. Khanb, K. Muhammad, W. Wuc, A. Ullah, S.W. Baik, Multi-grade brain tumor classification using deep CNN with extensive data augmentation, Journal of Computational Science, 30 (2019) 174-182.

[13] M.N. Tajik, A. Rehman, W. Khan, B. Khan, Texture feature selection using GA for classification of human brain MRI scans, In International Conference on Swarm Intelligence, (2016) 233-244).

[14] M. Toğaçar, Z. Cömert, B. Ergen, Classification of brain MRI using hyper column technique with convolutional neural network and feature selection method, Expert Systems with Applications, 149 (2020) 113274.

[15] A. Ari, D. Hanbay, Deep learning based brain tumor classification and detection system, Turkish Journal of Electrical Engineering \& Computer Sciences, 26:5 (2018) 2275-2286.

[16] Rembrandt The Cancer Imaging Archive, http://doi.org/10.7937/K9/TCIA.2015.588OZUZB

[17]D. Barboriak, Data from RIDER_NEURO_MRI, The Cancer ImagingArchive, (2015). http://doi.org/10.7937/K9/TCIA.2015.VOSN3HN1

[18] K.M. Schmainda, M. Prah, Data from Brain-Tumor-Progression The Cancer Imaging Archive, (2018) http://doi.org/10.7937/K9/TCIA.2018.15quzvnb

[19] K. Clark, B. Vendt, K. Smith, J. Freymann, J. Kirby, P. Koppel, S.Moore, S. Phillips, D. Maffitt, M. Pringle, L. Tarbox, F. Prior, The cancer imaging archive (TCIA): Maintaining and operating a public information repository, Journal of digital imaging, 26:6 (2013) 1045-1057.

[20] A. Krizhevsky, I. Sutskever, G.E. Hinton, ImageNet classification with deep convolutional neural networks. Communications of the ACM 60:6 (2017) 84-90.

[21]I. Kononenko, Estimating attributes: analysis and extensions of RELIEF. In European conference on machine learning, (1994) 171-182.

[22]L. Jin, Q. Zeng, J. He, Y. Feng, S. Zhou, Y. Wu, A ReliefF-SVM-based method for marking dopamine-based disease characteristics: A study on SWEDD and Parkinson's disease, Behavioural brain research, 356 (2019) 400-407.

[23] P. Kaur, G. Singh, P. Kaur, Classification and Validation of MRI Brain Tumor Using Optimised Machine Learning Approach, International Conference on Data Science, Machine Learning \& Applications, (2020) 172-189.

[24] A. Ari, N. Alpaslan, D. Hanbay, Computer-aided tumor detection system using brain MR images, Medical Technologies National Conference, (2015) 1-4.

[25] M. Ghasemi, M. Kelarestaghi, F. Eshghi, A. Sharifi, AFDL: a new adaptive fuzzy dictionary learning for medical image classification, Pattern Analysis and Applications, (2020) 1-20.

[26]J. Amin, M. Sharif, M. Yasmin, S.L. Fernandes, A distinctive approach in brain tumor detection and classification using MRI, Pattern Recognition Letters , (2017)

[27]B. Doğan, S.K. Çalık, Ö. Demir, Beyin Tümörlerinin Biçimsel Yapılandırma Kullanılarak Bilgisayar Destekli Tespiti, Uludağ University Journal of The Faculty of Engineering, 21:2 (2016) 257-268. 